\title{
Enhanced Adsorption Capacity of Ultralong Hydrogen Titanate Nanobelts for Antibiotics
}

\author{
Wenyao Li, ${ }^{a}$ Jingru Wang, ${ }^{a}$ Guanjie He, ${ }^{\mathrm{b}} \mathrm{Li}$ Yu, ${ }^{\mathrm{c}}$ Nuruzzaman Noor, ${ }^{\mathrm{d}}$ Yangang Sun, ${ }^{\mathrm{a}}$ Xiying Zhou, ${ }^{\text {a }}$ \\ Junqing $\mathrm{Hu}^{* \mathrm{e}}$ and Ivan P. Parkin*b \\ ${ }_{5}$ Received (in $\left.X X X, X X X\right)$ Xth $X X X X X X X X X 20 X X$, Accepted Xth XXXXXXXXX 20XX \\ DOI: 10.1039/b000000x
}

Limited by the relatively low adsorption capacity for antibiotics in inorganic nanomaterials, the ultralong hydrogen titanate nanobelts (UHTNs) with hollow structure and high surface area $\left(442.21 \mathrm{~m}^{2} \mathrm{~g}^{-1}\right)$ were synthesized to evaluate the feasibility as a potential adsorbent material for antibiotic removal. A batch of 10 adsorption experiments were conducted by using norfloxacin (NFO), tetracycline (TC) and ofloxacin (OFO) as the model antibiotic molecule. The results indicate that the adsorption of antibiotics on UHTNs are better fitted to the pseudo-second-order kinetics model, and the UHTNs maximum adsorption capacity calculated from Langmuir isotherm model was $151.51 \mathrm{mg} \mathrm{g}^{-1}$ for TC, $111.73 \mathrm{mg} \mathrm{g}^{-1}$ for NFO, and $148.14 \mathrm{mg} \mathrm{g}^{-1}$ for $\mathrm{OFO}$ at $\mathrm{PH}=7$, which is far better than most reported inorganic adsorbent

15 materials. In the adsorption process of tetracycline, the surface complexation between the adsorbent and TC contributed most to the adsorption, this has elucidated by Fourier Transform Infrared spectroscope (FT-IR) and X-ray photoelectron spectroscopy (XPS). In addition, because the UHTNs is up to tens of micrometers in length, it can be easily aggregated to form a network. Therefore, a novel paper-like, freestanding UHTNs membrane was fabricated via a simple vacuum filtration method, which also exhibits 20 good adsorption capacity.

\section{Introduction}

In the last decades, pharmaceutical antibiotics are used worldwide in human therapy, the livestock and the farming industry[1, 2]. ${ }_{25}$ Since most antibiotics including tetracycline (TC)[3], norfloxacin (NFO)[4] and ofloxacins (OFO)[5] are not fully metabolized in the body or eliminated in the natural environment, large fractions are excreted in its pharmaceutically active form [6]. These antibiotics are frequently detected in surface soil and water, ground-water, 30 sediments, and even drinking water. While, the existence of antibiotics in aquatic environment is recognized as a potential toxicological pollutant, which has a variety of potential adverse effects, such as acute and chronic toxicity, impact on aquatic photosynthetic organisms, disruption of indigenous microbial 35 populations, and the development of resistant bacterial strains [7, 8]. In turns, some more dangerous scenario, that may transport such strains to humans resulting in untreatable disease. Thus, the removal of antibiotics from water sources has been the subject of research interest over the last two decades[9].

40 Adsorption is a well-researched and operational facile technique because of its simplicity of use, high efficiency, and wide availability. It is observed that the adsorption effect is dependent on microstructure and surface functional group of antibiotics onto an adsorbent. Usually, antibiotics often contain various active
45 functional groups (such as $-\mathrm{COOH},-\mathrm{OH},-\mathrm{NH}_{2}$, etc.) and other electrostatic points containing heterogeneous atoms (-F, $-\mathrm{Cl}$, etc.). They adsorb onto a surface through an interactive mechanism or through a physically controlled mechanism [10]. Interactive sorption is the interaction of functional groups of adsorbent and 50 antibiotics species, such as van der Waals forces, electrostatic interactions, protonation, ion exchange, dipole-dipole interactions, $\mathrm{H}$-bonding, and complex-formation. The physical sorption is mainly dependent upon the mesoporous surface area of the adsorbent. Thus, some inorganic materials with high BET surface 55 area, porous texture, and mechanical stability have been considered a promising adsorbents for adsorbing antibiotics, such as bentonite clay [11], mesoporous $\mathrm{SiO}_{2}$ [12], $\mathrm{Al}_{2} \mathrm{O}_{3}$ [13], $\mathrm{Fe}_{2} \mathrm{O}_{3}$ [14], and halloysite nanotubes[15]. These inorganic adsorbents can afford enough contact area and make the physically controlled 60 mechanism played a great role in adsorbing pharmaceuticals. While the adsorption behavior is ineffective if only relies on physical sorption. In this scenario, adsorbents simultaneously possess large surface area and multiple functional groups are beneficial for their adsorptive capability of antibiotics.

${ }_{65}$ In this paper, UHTNs were synthesized for effective adsorption of antibiotics from the contaminated water. The UHTNs not only possess high surface area $\left(442.21 \mathrm{~m}^{2} \mathrm{~g}^{-1}\right)$ with hollow structure providing high contact area, but also contain abundant functional groups (Ti-OH, Ti-O...H-O-Ti, Ti-O-Ti). These groups have 
significant contribution towards the adsorption ability interacting with antibiotics. A batch of adsorption experiments were conducted by using norfloxacin (NFO), tetracycline(TC) and ofloxacin (OFO) as the model antibiotic molecule. The results 5 indicate that the adsorption of antibiotics on UHTNs better fitted a pseudo-second-order kinetics model, and the maximum adsorption capacity of the UHTNs calculated from Langmuir isotherm model was $151.51 \mathrm{mg} \mathrm{g}^{-1}$ for TC, $111.73 \mathrm{mg} \mathrm{g}^{-1}$ for NFO, and $148.14 \mathrm{mg}$ $\mathrm{g}^{-1}$ for $\mathrm{OFO}$ at $\mathrm{PH}=7$, which is significantly better than many 10 reported inorganic adsorbent materials. Moreover, to the best of our knowledge, this is the first to report that the UHTNs can serve as promising materials with enhanced adsorption of antibiotics from the contaminated water among various inorganic adsorbent materials.

\section{Experimental Section}

\subsection{Synthesis of the ultralong hydrogen titanate nanobelts}

The raw materials were an anatase and rutile two-phase mixture of nanopowders which was prepared by hydrolyzing titanium 20 tetrachloride[19]. In the optimized synthesis, $0.5 \mathrm{~g}$ of synthesized two-phase nanopowder was dispersed into $40 \mathrm{~mL} \mathrm{NaOH}$ solution $(10 \mathrm{M})$, and then transferred into a $50 \mathrm{~mL}$ Teflon-lined stainlesssteel autoclave with a magnetic rotor. The autoclave was put inside a methyl silicone oil bath on a magnetically stirred heating plate, 25 and the reaction was set at $120{ }^{\circ} \mathrm{C}$ for $48 \mathrm{~h}$. After reaction, the autoclave was taken from the oil bath and allowed to cool naturally to room temperature. The product was collected by centrifugation and washed several times with deionized water. After that, the wet centrifuged products were washed with $0.1 \mathrm{M} \mathrm{HNO}_{3}$ solution for

$3012 \mathrm{~h}$ to complete the hydrogen ion exchange process. Afterwards, the suspension was centrifuged and washed with deionized water several times until a $\mathrm{pH}$ value of 7 was reached. Finally, the products were dried in vacuum oven at $60{ }^{\circ} \mathrm{C}$ for $4 \mathrm{~h}$.

\subsection{Membrane fabrication of the ultralong hydrogen titanate 35 nanobelts}

Paper-like UHTNs membrane was constructed by a simple process of suction filtration. The first step is to collect the products which were synthetized by the above processes but did not undergo the drying process. Then, the sample was added to a certain amount 40 of deionized water under $30 \mathrm{~min}$ stirring to form a mixed solution. After that, this mixed solution was filtered under vacuum on a microfiltration Mixed Cellulose Esters Membrane (MCEM, pore size of $0.22 \mu \mathrm{m}$ ). Finally, the membranes were dried in a vacuum oven at $60{ }^{\circ} \mathrm{C}$ for $3 \mathrm{~h}$, and the UHTNs membrane detached from 45 the MCEM easily.

\subsection{Material Characterizations}

As-prepared products were characterized with a D/max-2550 PC X-ray diffractometer (XRD, Rigaku, $\mathrm{Cu}-\mathrm{K} \alpha$ radiation), scanning electron microscope (SEM, S-4800), and transmission ${ }_{50}$ electron microscope (TEM, JEM-2100F). The Brunauer-EmmettTeller (BET) specific surface area, pore volume, and pore size were calculated from $\mathrm{N}_{2}$ adsorption/desorption isotherms determined at $-196{ }^{\circ} \mathrm{C}$ using an automated nitrogen adsorption analyzer (ASAP 2020, Micromeritics, America). The surface 55 chemical structure of the UHTNs and TC were investigated by the
Fourier Transform Infrared spectrum (FT-IR) spectrometer (6700, Nicolet, USA). X-ray photoelectron spectroscopy (XPS) spectra were collected using a PHI Quantum-2000 electron spectrometer (Ulvac-Phi, Japan) with $150 \mathrm{~W}$ monochromatized $\mathrm{Al} \mathrm{K \alpha}$ radiation $60(1486.6 \mathrm{eV})$.

\subsection{Adsorption experiments setup and measurements}

All the adsorption experiments were conducted in $100 \mathrm{~mL}$ glass vials containing $5 \mathrm{mg}$ of the UHTNs sample as adsorbent. To obtain adsorption isotherms, $50 \mathrm{~mL}$ TC solutions with increasing 65 concentrations $\left(4-40 \mathrm{mg} \mathrm{L}^{-1}\right.$ ) were placed in contact with $5 \mathrm{mg}$ of the UHTNs adsorbent. The mixed solution was stirred for $20 \mathrm{~h}$ to ensure the adsorption-desorption equilibrium between the adsorbent and TC had established. During the adsorption process, the mixed solution was taken at regular intervals by using a $10 \mathrm{ml}$

70 plastic syringe and then filtered in a $0.22-\mu \mathrm{m}$ filter membrane. TC concentrations before and after adsorption were determined by a UV-vis spectrophotometer at $357 \mathrm{~nm}$. The amount of adsorbed TC $\left(q_{e}, \mathrm{mg} \mathrm{g}^{-1}\right)$ was calculated from the mass balance equation:

$$
q_{e}=\frac{\left(C_{0}-C_{e}\right) V}{m}
$$

75 where $C_{o}$ and $C_{e}\left(\mathrm{mg} \mathrm{L}^{-1}\right)$ are at initial and equilibrium concentration of TC, respectively. $V$ is the initial volume of the solution $(\mathrm{L})$ and $\mathrm{m}(\mathrm{g})$ is the mass of the UHTNs adsorbent. For comparison, such experiments were also carried out on two different antibiotics solutions, i.e., norfloxacin (NFO) and 80 ofloxacin (OFO).

\section{Results and Discussion}

\subsection{Structure and morphology of the ultralong hydrogen titanate nanobelts}

The as-synthesized product was characterized by XRD to 85 identify its crystallographic structure. As can be seen from Fig. 1, the main diffraction peaks at $2 \theta=9.78^{\circ}, 19.5^{\circ}, 24.1^{\circ}, 27.82^{\circ}, 48^{\circ}$, $49.2^{\circ}, 61.9^{\circ}, 62.8^{\circ}$ can be respectively denoted as the (200), (400), (110), (310), (020), (220), (002) and (202) reflections, these indexed to an orthorhombic phase of $\mathrm{H}_{2} \mathrm{Ti}_{2} \mathrm{O}_{5} \cdot \mathrm{H}_{2} \mathrm{O}$ (JCPDS Card 90 No. 47-0124).

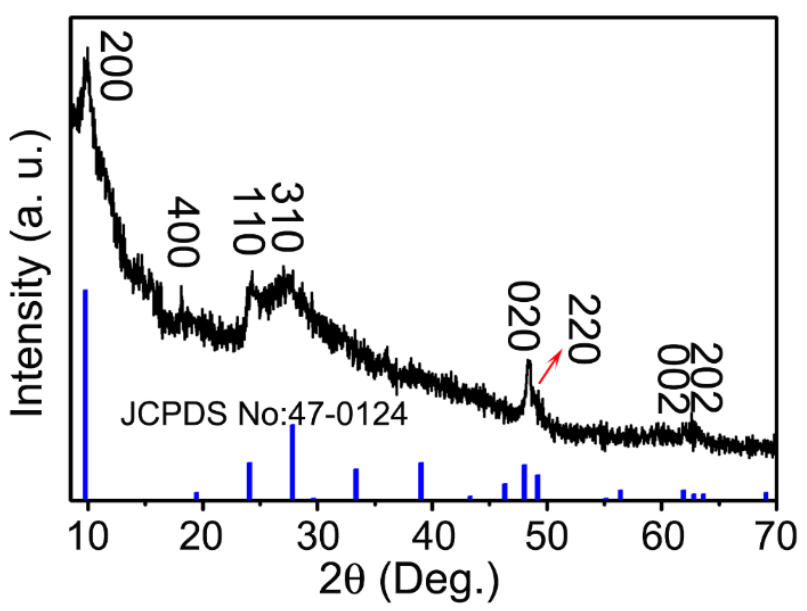

Fig. 1 XRD pattern of the ultralong hydrogen titanate nanobelts

The microstructure of the $\mathrm{H}_{2} \mathrm{Ti}_{2} \mathrm{O}_{5} \cdot \mathrm{H}_{2} \mathrm{O}$ was then studied by SEM and TEM. The SEM image in Fig. 2a indicates that the 
products are consisted of a large quantity of twisted nanobelts with length up to tens of micrometres. The inset shows an optical image of the white membrane prepared by the UHTNs (see Experimental Section). Interestingly, after carefully examination by TEM, it was 5 observed that the middle part of the nanobelts seems to have a hollow structure, as shown in Fig. 2b. The high-magnification TEM investigation (Fig. 2c) indicates that the typical inner diameter of the nanobelts is $\sim 18 \mathrm{~nm}$, and the thickness of the wall is $\sim 8 \mathrm{~nm}$. Further, the high-resolution TEM image showed in Fig. ${ }_{10} 2 \mathrm{~d}$ reveals that they are structurally uniform single crystals and no amorphous sheathed phases formed on the surface. The lattice fringes give an interplanar spacing of $0.18 \mathrm{~nm}$ (the bottom left inset), corresponding to the (220) lattice plane of the orthorhombic phase $\mathrm{H}_{2} \mathrm{Ti}_{2} \mathrm{O}_{5} \cdot \mathrm{H}_{2} \mathrm{O}$, which agree well with the XRD pattern 15 results. To further investigate the detailed structure, the corresponding FFT diffraction pattern (the upper right inset) was also taken from the HRTEM image given in Fig. 2d, which also indicates the crystalline nature of the nanobelts.

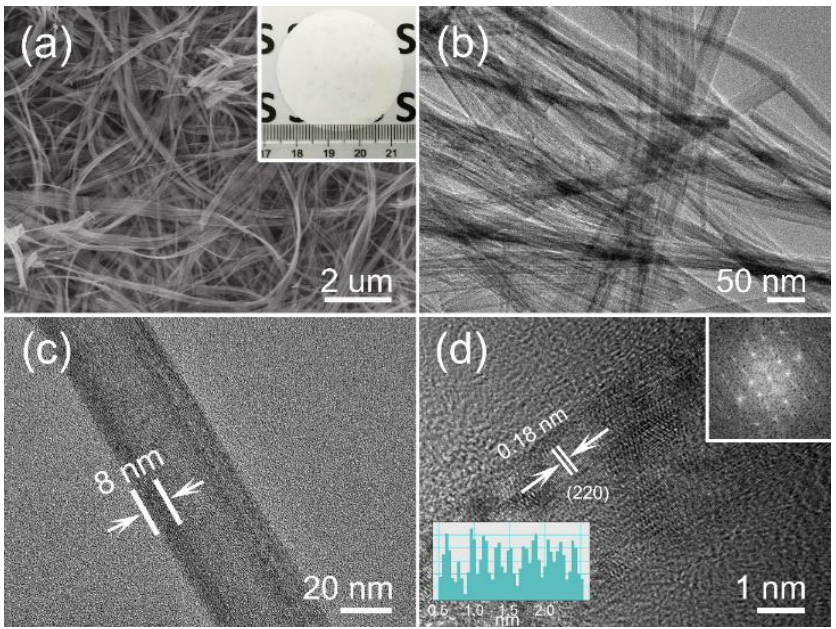

20 Fig. 2 (a-d) SEM, Low- and High-magnification TEM, and HRTEM images of the ultralong hydrogen titanate nanobelts, respectively. Inset of (a) shows an optical image of a membrane made from the nanobelts, insets of (d) show the corresponding lattice (bottom left) and FFT pattern (upper right), respectively.

\section{${ }_{25} 3.2$ BET and pore size of the ultralong hydrogen titanate nanobelts}

The microstructure of the ultralong hydrogen titanate nanobelts was further investigated by the $\mathrm{N}_{2}$ adsorption-desorption isotherms, and the corresponding results are presented in Fig. 3, 30 the specific surface area of UHTNs is as large as $442.21 \mathrm{~m}^{2} \mathrm{~g}^{-1}$. As can be seen from Fig. 3, the $\mathrm{N}_{2}$ adsorption-desorption isotherms of the UHTNs assigned to typical type IV adsorption isotherm, which clearly indicate the presence of a mesoporous structure. In addition, the hysteresis loop of the isotherm belongs to a type $\mathrm{H} 3$ 35 hysteresis loop, which suggests the presence of slit-shaped pores. From the pore size distribution (inset of Fig. 3), the pore radius is centered at $\sim 18 \mathrm{~nm}$, agreed well with the inner diameter of the hollow nanobelts characterized from TEM image shown in Fig. 2c. Besides, the small peak intensity centered at $\sim 5 \mathrm{~nm}$ could attributed 40 to the assembly of the nanobelts.

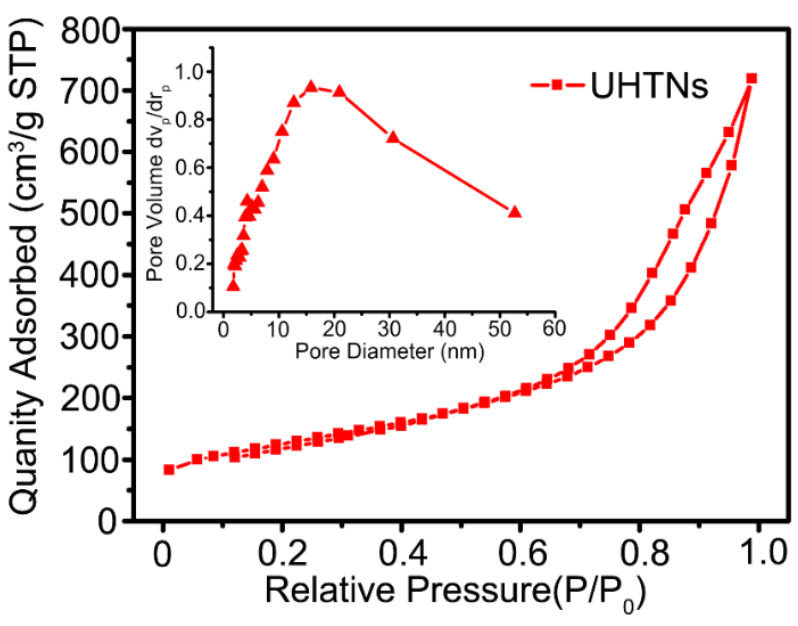

Fig. 3 The nitrogen adsorption-desorption isotherm of the ultralong hydrogen titanate nanobelts. The inset shows the corresponding pore size distributions.

\section{${ }_{45}$ 3.3 Adsorption performance of the ultralong hydrogen titanate nanobelts}

\subsubsection{Equilibrium adsorption isotherms}

The antibiotics TC, NFO and OFO were separately adsorbed onto UHTNs and allowed to equilibrate under different 50 concentrations as depicted in Fig. 4. Two typical adsorption models (the Langmuir and Freundlich models) were used to fit the experimental equilibrium adsorption data. The Langmuir and Freundlich models[20] are respectively expressed as follows:

$$
\begin{aligned}
\frac{C_{e}}{q_{e}} & =\frac{1}{K_{L} q_{m}}+\frac{C_{e}}{q_{m}} \\
\ln q_{e} & =\ln K_{f}+\left(\frac{1}{n}\right) \ln C_{e}
\end{aligned}
$$

where $q_{m}\left(\mathrm{mg} \mathrm{g}^{-1}\right)$ is the maximum adsorption capacity and $K_{L}$ $\left(\mathrm{L} \mathrm{mg}^{-1}\right)$ is the Langmuir adsorption equilibrium constant. $K_{f}((\mathrm{mg}$ $\left.\left.\mathrm{g}^{-1}\right)\left(\mathrm{L} \mathrm{mg} \mathrm{m}^{-1}\right)^{1 / \mathrm{n}}\right)$ and $\mathrm{n}$ are Freundlich constant and adsorption intensity (dimensionless) of the adsorbents, respectively. (a)

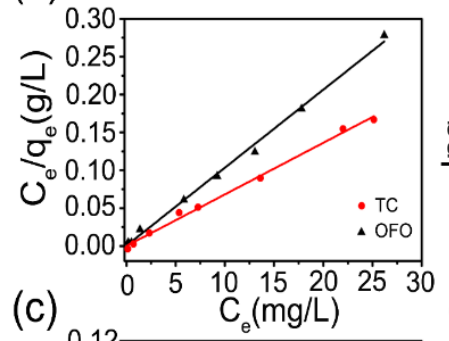

(c)

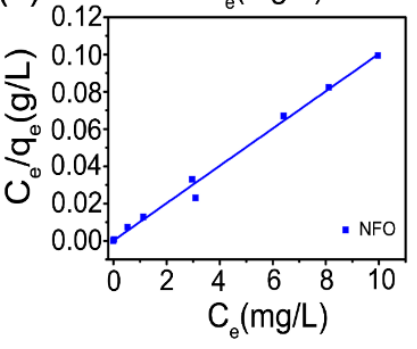

(b)
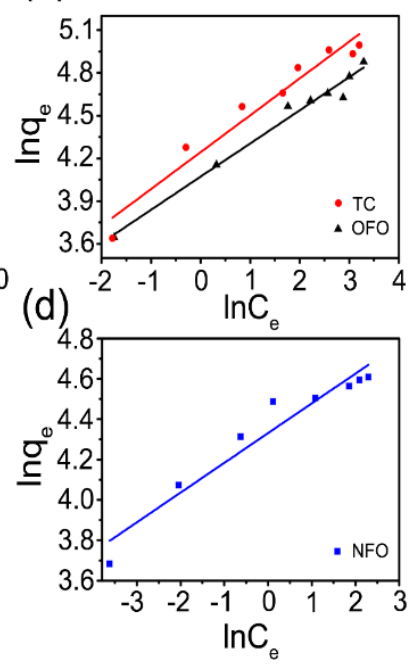

Fig. 4 The linear fitting curves of Langmuir (a, c) and Freundlich (b, d) models for UHTNs towards TC, NFO and OFO. 
The Langmuir and Freundlich fittings are shown in Fig. 4, and the regression data for the two models are listed in Tab. 1. For UHTNs, both Langmuir and Freundlich equations describe the adsorption isotherm well, however the former is better. All the 5 fitting correlation coefficients $\left(\mathrm{R}^{2}\right)$ of antibiotics adsorption form Langmuir model were higher than 0.9836 (shown in Tab. 1). This indicates that the adsorption process corresponds to a homogeneous surface and signal-molecular layer adsorption for the synthesized materials. Meanwhile, the $n$ values were all larger 10 than 1.0, suggesting favorable adsorption. initial period of $10 \mathrm{~min}$, followed by slightly increase with incubation time. It nearly reaches a plateau after approximately 60 min. While, the adsorption of TC and NFO on UHTNs required 30 nearly $\sim 100 \mathrm{~min}$ to reach a plateau. The kinetics were fitted by pseudo-first-order and pseudo-second order models[25]. The mathematical forms of these models are given as follows, respectively:

35

$$
\begin{gathered}
\ln \left(q_{e}-q_{t}\right)=\ln q_{e}-k_{1} t \\
\frac{t}{q_{t}}=\frac{1}{k_{2} q_{e}^{2}}+\frac{t}{q_{e}}
\end{gathered}
$$

\begin{tabular}{|c|c|c|c|c|c|c|}
\hline \multirow{2}{*}{$\begin{array}{c}\text { Isotherm models } \\
\text { Pollutants }\end{array}$} & \multicolumn{3}{|c|}{ Langmuir } & \multicolumn{3}{|c|}{ Freundlich } \\
\hline & $\mathrm{k}_{\mathrm{L}}\left(\mathrm{L} \mathrm{mg}^{-1}\right)$ & $\mathrm{q}_{\mathrm{m}}\left(\mathrm{mg} \mathrm{g}^{-1}\right)$ & $\mathrm{R}^{2}$ & $\mathrm{k}_{\mathrm{f}}\left(\mathrm{mg} \mathrm{g}^{-1}\right)\left(\mathrm{L} \mathrm{mg}^{-1}\right)^{1 / n}$ & $\mathrm{n}$ & $\mathrm{R}^{2}$ \\
\hline$\overline{\mathrm{TC}}$ & 0.8333 & 151.51 & 0.9947 & 69.6728 & 3.8667 & 0.9451 \\
\hline NFO & 0.9391 & 111.73 & 0.999 & 76.0043 & 6.7916 & 0.9253 \\
\hline $\mathrm{OFO}$ & 0.2412 & 148.14 & 0.9836 & 58.6548 & 4.2990 & 0.9766 \\
\hline
\end{tabular}

Tab. 1 Langmuir and Freundlich regression data from the adsorption isotherms of TC, OFO and NFO by the UHTNs.

Tab. 2 The adsorption capacity of antibiotics by currently available adsorbents.

\begin{tabular}{ccccc}
\hline Antibiotics & Adsorbents & $\mathrm{q}_{\max }\left(\mathrm{mg} \mathrm{g}^{-1}\right)$ & Condition & Ref. \\
\hline \multirow{5}{*}{ Tetracycline } & Alumina & 53.73 & $25^{\circ} \mathrm{C} \mathrm{PH}=7$ & {$[13]$} \\
& Na-montmorillonite & 33 & $25^{\circ} \mathrm{C} \mathrm{PH}=5.5$ & {$[11]$} \\
& Halloysite nanotubes & 34.2 & $25^{\circ} \mathrm{C} \mathrm{PH=4-7}$ & {$[15]$} \\
& Kaolinite & 3.8 & $25^{\circ} \mathrm{C} \mathrm{PH}=4$ & {$[21]$} \\
& Mesoporous silica & 44.4 & $23^{\circ} \mathrm{C} \mathrm{PH}=6$ & {$[22]$} \\
Ofloxacin & UHTNs & 151.51 & $25^{\circ} \mathrm{C} \mathrm{PH=7}$ & This study \\
& Nonporous $\mathrm{SiO}_{2}$ & 18.7 & $25^{\circ} \mathrm{C} \mathrm{PH=7.2}$ & {$[23]$} \\
Norfloxacin & UHTNs & 148.14 & $25^{\circ} \mathrm{C} \mathrm{PH=7}$ & This study \\
& Alumina pure & 6.4 & $22-25^{\circ} \mathrm{C} \mathrm{PH}=6.8$ & {$[24]$} \\
& UHTNs & 111.73 & $25^{\circ} \mathrm{C} \mathrm{PH=7}$ & This study \\
\hline
\end{tabular}

The order of $q_{m}$ for the three antibiotics adsorbed by UHTNs based on the Langmuir isotherms was TC $\left(151.51 \mathrm{mg} \mathrm{g}^{-1}\right)>$ OFO $\left(148.14 \mathrm{mg} \mathrm{g}^{-1}\right)>$ NFO (111.73 $\left.\mathrm{mg} \mathrm{g}^{-1}\right)$. Significantly, these capacities are more than 3 times higher than those of previously 15 reported inorganic adsorbent materials in the literature, as shown in Tab. 2. In addition, the membrane fabricated by UHTNs is also tested for adsorption of the TC, the adsorption capacity can reach $133.3 \mathrm{mg} \mathrm{g}^{-1}$. The results showed that UHTNs were effective in removing these sets of antibiotic from aqueous solution.

\section{3.3.2 Adsorption kinetics}

The adsorption behaviors of the UHTNs were then studied by the adsorption-desorption equilibrium tests. Fig. 5a shows the adsorption kinetics of the samples for different initial concentrations of TC (40 mg L-1), OFO (40 mg L-1) and NFO (20 $\left.25 \mathrm{mg} \mathrm{L}^{-1}\right)$. The equilibrium was reached after around $3 \mathrm{~h}$ of incubation. The adsorption of OFO on UHTNs was fast during the where $q_{e}\left(\mathrm{mg} \mathrm{g}^{-1}\right)$ and $q_{t}\left(\mathrm{mg} \mathrm{g}^{-1}\right)$ are the amounts of adsorbed by the adsorbents at equilibrium and the adsorptive time $t$ (min), respectively. $K_{I}$ is the rate constant of the pseudo-first-order kinetic model $\left(\mathrm{min}^{-1}\right)$. The values of $q_{e}$ and $K_{l}$ can be determined 40 from the intercept and slope of the linear plots of $\ln \left(q_{e^{-}} q_{t}\right) v s$. $t$, respectively. $K_{2}\left(\mathrm{~g} \mathrm{mg}^{-1} \mathrm{~min}^{-1}\right)$ is the rate constant of the pseudosecond-order kinetic model for adsorption. The straight-line plots of $t / q_{t} v s$. $t$ were tested to obtain the rate parameters. The results of kinetics data analysis are summarized in Tab. 3.

45 The intra-particle diffusion model is represented as follows [25]:

$$
q_{t}=k_{i d} t^{1 / 2}+C
$$

where $C\left(\mathrm{mg} \mathrm{g}^{-1}\right)$ is the intercept and $k_{\text {id }}\left(\mathrm{mg} \mathrm{g}^{-1} \mathrm{~min}^{-1 / 2}\right)$ is the intra-particle diffusion rate constant, which can be calculated from the slope of the linear plots of $q_{\mathrm{t}} v s . t^{1 / 2}$.

50 Linear regressions of the kinetic plots are shown in Fig. $5 \mathrm{~b}$ and 5c. The kinetic parameters and calculated initial adsorption rates

\begin{tabular}{|c|c|c|c|c|c|c|c|c|}
\hline \multirow{2}{*}{ Model } & \multirow{2}{*}{$\begin{array}{l}\mathbf{q}_{\mathbf{e}}, \exp \\
(\mathrm{mg} / \mathrm{g})\end{array}$} & \multirow{2}{*}{$\mathbf{C}_{0}\left(\mathrm{mg} \mathrm{L}^{-1}\right)$} & \multicolumn{3}{|c|}{ Pseudo-first-order } & \multicolumn{3}{|c|}{ Pseudo-second-order } \\
\hline & & & $\mathrm{K}_{1}\left(\mathrm{~L} \mathrm{~min} \min ^{-1}\right)$ & $\mathrm{q}_{\mathrm{e}, \mathrm{cal}}\left(\mathrm{mg} \mathrm{g}^{-1}\right)$ & $\overline{\mathrm{R}^{2}}$ & $\mathrm{~K}_{2}\left(\mathrm{~g} \mathrm{mg}^{-1} \mathrm{~min}^{-1}\right)$ & $\mathrm{q}_{\mathrm{e}, \text { cal }}\left(\mathrm{mg} \mathrm{g}^{-1}\right)$ & $\overline{\mathrm{R}^{2}}$ \\
\hline $\mathrm{TC}$ & 92.16 & 40 & 0.02454 & 70.4582 & 0.98503 & $6.6678 \times 10^{-4}$ & 99.01 & 0.992 \\
\hline NFO & 91.46 & 40 & 0.01395 & 67.1085 & 0.91429 & $9.1145 \times 10^{-4}$ & 96.2464 & 0.999 \\
\hline OFO & 67.31 & 20 & 0.02643 & 13.7374 & 0.97745 & $3.7919 \times 10^{-4}$ & 67.3401 & 0.995 \\
\hline
\end{tabular}
are listed in Tab. 3. Based on the correlation coefficients $\left(\mathrm{R}^{2}\right)$, the

Tab. 3 Kinetic parameters of TC, NFO, OFO adsorption 
experimental data are best described by a pseudo-second-order model. Moreover, the theoretical adsorption capacity values $\left(q_{\mathrm{e}, \mathrm{cal}}\right)$ were all consistent with the experimental adsorption capacity values $\left(q_{\mathrm{e}, \exp }\right)$ by using this model. The results showed that the 5 pseudo-second-order model was the best model for representation of UHTNs kinetics data.

Because the pseudo-second-order model cannot provide sufficient information about the diffusion mechanism during the adsorption process, the intra-particle diffusion model was used to 10 determine the adsorption process of TC, OFO, NFO onto UHTNs. The fitting plots of $q_{\mathrm{t}}$ versus $t^{1 / 2}$ for TC, OFO and NFO are shown in Fig. 5d. This shows three different stages during the adsorption process[26]: (1) external mass transfer of the adsorbate across the liquid film to the adsorbent exterior surface, which is also termed 15 the "outer diffusion" (or boundary layer diffusion); (2) transport of the adsorbate from the adsorbent exterior surface to the pores or capillaries of the adsorbent internal structure, which is termed the "intra-particle diffusion" (or inner diffusion); and (3) adsorption of the adsorbate onto the active sites on the inner and outer surfaces 20 of the adsorbent. The third step is considered very fast and thus cannot be treated as the rate-controlling step and was considered as negligible. The regression of $q_{\mathrm{t}} v s . t^{1 / 2}$ for antibiotic solution was linear and did not pass through the origin, indicating that the boundary layer diffusion was in control during the adsorption 25 process and the intra-particle diffusion was involved in the adsorption process, however, it is not the sole rate-limiting step. (a)
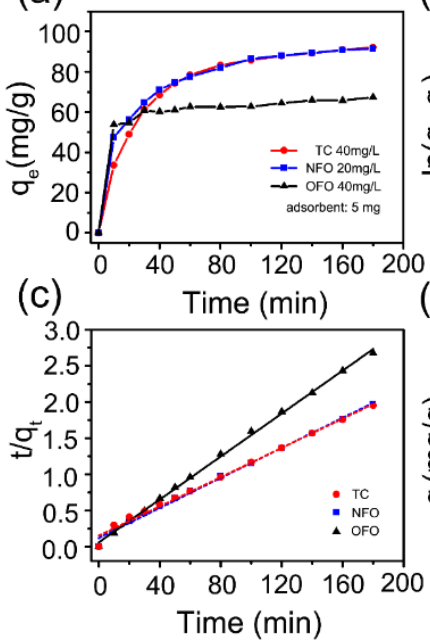

(d)
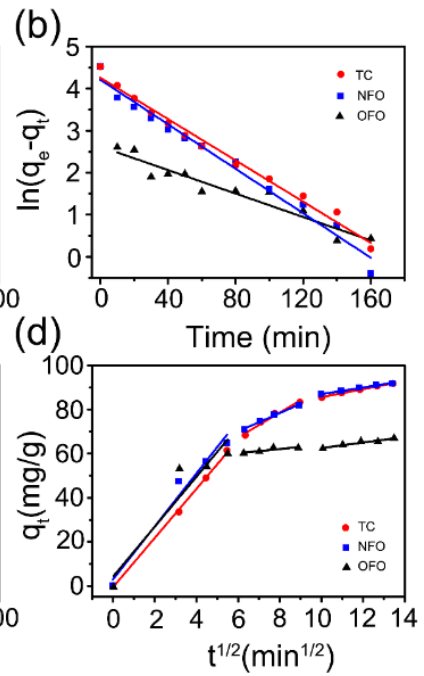

Fig. 5 (a) Adsorption kinetics of TC, NFO, OFO on UHTNs. Linear regression kinetics plots: (b) pseudo-first-order model; (c) pseudo-second30 order model; (d) intra-particle-diffusion model.

\subsubsection{Adsorption mechanism}

Based on the above analysis, the UHTNs show the best adsorption performances towards TC. In order to understand the detailed adsorption mechanism, the functional groups of UHTNs 35 samples before and after adsorption were firstly analyzed by FTIR spectroscopy, as shown in Fig. 6a. Before the adsorption, the UHTNs spectrum presents a broad band centered at $400 \sim 800 \mathrm{~cm}^{-1}$ due to the vibration of the Ti-O bonds. The small peak at $1633 \mathrm{~cm}^{-}$

${ }^{1}$ and the broad peak at $3369 \mathrm{~cm}^{-1}$ can be attributed to the $-\mathrm{OH}$ 40 groups bending and stretching vibration that reported in the literature [27]. A small peak appeared at $1384 \mathrm{~cm}^{-1}$ that belonged to the N-O groups which was brought by the nitric acid in the acid washing process. After the adsorption, the intensity of peaks corresponding to - $\mathrm{OH}$ vibrations were found to become weaker and 45 some peaks shifts were noted from the initial positions, which is due to the successful adsorption of TC. For further investigation, the FT-IR spectroscopy of pure TC was also carried out. In the spectrum of pure TC, characteristic peaks due to amide groups were present at 1607 and $1586 \mathrm{~cm}^{-1}$. The peak at $1228 \mathrm{~cm}^{-1}$ 50 correspond to $\mathrm{C}-\mathrm{N}$ amide vibrations [15]. After complexation with UHTNs, the bands were noted to be shifted. Meanwhile, the relatively strong peak appeared at $460 \mathrm{~cm}^{-1}$ come from residue groups of TC degradation. All of this conformed the participation of amide group for the adsorption of TC, and the UHTNs can 55 adsorb the TC effectively.

XPS analysis of UHTNs after the adsorption of TC was carried out to further investigate the interactions between TC and the UHTNs. Fig. $6 \mathrm{~b}$ gives the wide survey scan of XPS spectra in the range of $0-1350 \mathrm{eV}$. No extra peak other than those corresponding 60 to $\mathrm{C}, \mathrm{N}, \mathrm{Ti}$, and $\mathrm{O}$ were observed in the spectrum, indicating that no impurities are formed. The high resolution XPS spectra of Ti $2 p$ region of the samples display a typical core level spectra of Ti-O consisting of two broad peaks positioned at 458.5 and $464.2 \mathrm{eV}$ (Fig. 6c). The O 1s XPS spectrum of UHTNs after adsorption of ${ }_{65} \mathrm{TC}$ can be deconvoluted into three individual component peaks located at 529.9, 530.5 and 531.6 eV (Fig. 6d), which are attributed to lattice oxygen in the metal oxide (Ti-O), carbon-oxygen (C-O) bonds from TC, and oxygen atoms present as surface hydroxyl groups (H-O), respectively. The quantitative calculation results 70 show that the atomic ratio of $\mathrm{Ti} / \mathrm{O}$ was 0.515 , which means a lot of $\mathrm{O}$ atoms were consumed and formed some complexation in the adsorption process. The relative contents of oxygen containing groups in the UHTNs were also calculated and summarized in Tab. S1 (ESI $\dagger$ in supporting information). After adsorption, some active $75 \mathrm{O}$ atoms were decreased and form new chemical bonds. The relative content of $\mathrm{O}-\mathrm{H}$ was just $15.57 \%$. Meanwhile, the proportion of the Ti-O bonds was up to $58.33 \%$ implied that the adsorption mechanism was mainly ascribed to the substitution of Ti-OH groups by oxygen containing functional groups of TC. (a)

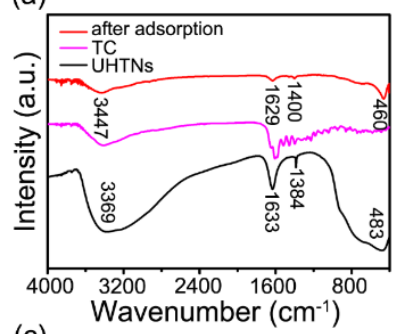

(c)

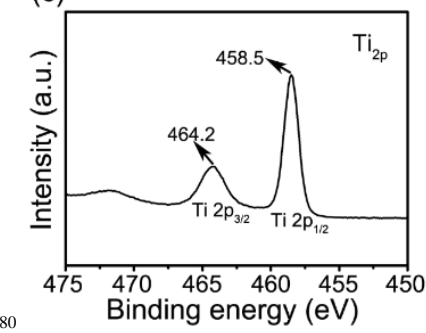

(b)

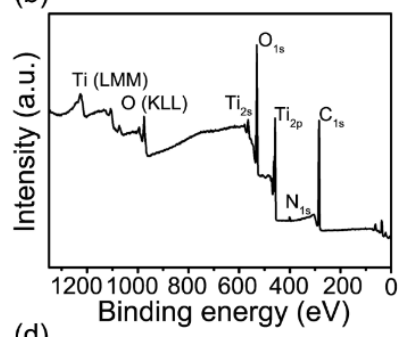

(d)

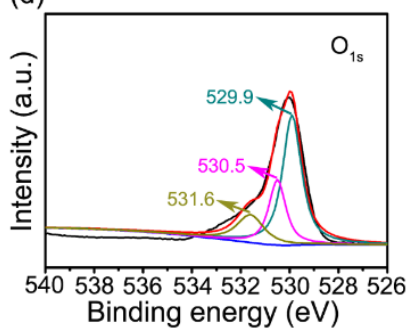

Fig. 6 (a) FT-IR spectra of TC and UHTNs samples before and after adsorption; (b) XPS wide-scan spectrum, high resolution XPS spectra of (c) Ti $2 \mathrm{p}$ and (d) $\mathrm{O} 1 \mathrm{~s}$ after adsorption of TC. 


\section{Conclusions}

In summary, ultralong hydrogen titanate nanobelts $\left(\mathrm{H}_{2} \mathrm{Ti}_{2} \mathrm{O}_{5} \cdot \mathrm{H}_{2} \mathrm{O}\right.$, UHTNs) were synthesized for effective adsorption of antibiotics from the contaminated water. The hollow structure 5 of the as-synthesized nanobelts endow the material with high porosity with an average inner diameter of $18 \mathrm{~nm}$, and a BET surface area of up to $442.21 \mathrm{~m}^{2} / \mathrm{g}$. As an adsorbent, the UHTNs can remove TC, OFO, and NFO from aqueous solution efficiently, with the maximum adsorption capacity (Langmuir isotherm) of $10151.52 \mathrm{mg} \mathrm{g}^{-1}$ for $\mathrm{TC}$, which is comparatively high among the reported inorganic adsorbent materials. In addition, the underlying mechanism of TC adsorption was elucidated by FTIR and XPS analysis. The surface complexation between TC and the surface hydroxyl group of the nanobelts was a dominant interaction to 15 boost the adsorption capacity. Given the worldwide pharmaceutical antibiotics pollution, this study may prove a useful design of adsorbents in environmental remediation.

\section{Acknowledgements}

This work was financially supported by the National Natural ${ }_{20}$ Science Foundation of China (Grant No. 51602193), the China Scholarship Council/University College London for the Joint PhD scholarship program, the Foundation of Shanghai University of Engineering Science (2015-05) and the project of Shanghai universities young teacher training scheme (ZZGCD15037).

\section{${ }_{25}$ Notes and references}

${ }^{a}$ School of material engineering, Shanghai university of engineering science, Shanghai 201620, China.

${ }^{b}$ Materials Chemistry Centre, Department of Chemistry, University College London, 20 Gordon Street, London WC1H OAJ, U. K.

${ }_{30}{ }^{c}$ Shenzhen Key Laboratory of Laser Engineering, Key Laboratory of Optoelectronic Devices and Systems of Ministry of Education and Guangdong Province, College of Optoelectronic Engineering, Shenzhen University, Shenzhen 518060, China

${ }^{d}$ Institute of Textiles and Clothing Q Core, 7/F The Hong Kong Polytechnic 35 University Hunghom, Kowloon, Hong Kong

${ }^{e}$ State Key Laboratory for Modification of Chemical Fibers and Polymer Materials, College of Materials Science and Engineering, Donghua University, Shanghai 201620, China.

Fax: +86-21-6779-2947; Tel: +86-21-6779-2947; E-mail:

40 i.p.parkin@ucl.ac.uk; hu.junqing@dhu.edu.cn; zhouxiying@sues.edu.cn $\dagger$ Electronic Supplementary Information (ESI) available: [Experimental process, Supplementary Fig.s and Specific capacitance calculation]. See DOI: $10.1039 / \mathrm{b} 000000 \mathrm{x} /$
1 X. Chang, M. T. Meyer, X. Liu, Q. Zhao, H. Chen, J. A. Chen, Z. Qiu,

45 L.Yang, J. Cao, W. Shu, Environ. Pollut. 2010, 158, 1444.

2 V. Homem, L.Santos, J. Environ. Manage. 2011, 92, 2304.

3 B. Luo, D. Xu, D. Li, G. Wu, M. Wu, W. Shi, ACS Appl. Mater. Inter. 2015, 7, 17061.

4 M. Chen, W. Chu, Ind. Eng. Chem. Res. 2012, 51, 4887.

505 H. Peng, B. Pan, M. Wu, Y. Liu, D. Zhang, B. Xing, J. Hazard Mater. 2012, 233, 89 .

6 G. Rigos, I. Nengas, M. Alexis, G. M. Troisi, Aquat. Toxicol. 2004, 69, 281.

7 C. L. Wong, Y. N. Tan, A. R.Mohamed, J. Environ. Manage. 2011, 92, 1669.

8 M. Addamo, V. Augugliaro, A. D. Paola, E. García-López, V. Loddo, G. Marcì, L. Palmisano, J. Appl. Electrochem. 2005, 35, 765.

9 N. Suriyanon, P. Punyapalakul, C. Ngamcharussrivichai, Chem. Eng. J. 2013, 214, 208.

6010 J. Akhtar, N. A. S.Amin, K. Shahzad, Desalin. .Water Treat. 2015, 57, 12842.

11 A. Raquel, A. L. FigueroaI, A. Allison, Environ.Sci.Technol. 2004, 38, 476.

12 Z. Liang, Z. Zhao, T. Sun, W. Shi, F. Cui, J. Hazard Mater. 2016, 305, 658.

13 B. Zhao, Y. Ji. F. Wang, H. Lei, Z. Gu, Desalin. Water Treat. 2015, 57, 5174.

14 H. Wu, H. Xie, G. He, Y. Guan, Y. Zhang, Appl. Clay Sci. 2016, 119,161.

7015 W. T. Jiang, P.-H. Chang, Y. Tsai, Z. Li, Micropor. Mesopor. Mat. 2016, 220, 29.

16 Y. Tang, Y. Zhang, J. Deng, J. Wei, H. Le Tam, B. K. Chandran, Z. Dong, Z. Chen, X. Chen, Adv. Mater. 2014, 26, 6111.

17 Y. Tang, Y. Zhang, J. Deng, D. Qi, W. R. Leow, J. Wei, S. Yin, Z.

75 Dong, R.Yazami, Z. Chen, X. Chen, Angew. Chem. Int. Edit. 2014, $\mathbf{5 3}, 13488$.

18 L. Li, Y. Li, C. Yang, Carbohyd. Polym. 2016, 140, 299.

19 S. Novaconi, N. Vaszilcsin, Mater. Lett. 2013, 95, 59.

20 J. Dai, J. He, A. Xie, L. Gao, J. Pan, X. Chen, Z. Zhou, X. Wei, Y. Yan, Chem. Eng. J. 2016, 284, 812.

21 Z. Li, L. Schulz, C. Ackley, N. Fenske, J. Colloid Interf. Sci. 2010, 351, 254.

22 I. Turku, T. Sainio, E. Paatero, Environ. Chem. Lett. 2007, 5, 225.

23 K. W. Goyne, J. Chorover, J. D. Kubicki, A. R. Zimmerman, S. L. 85 Brantley, J. Colloid Interf. Sci. 2005, 283, 160.

24 O. Lorphensri, J. Intravijit, D. A. Sabatini, T. C. Kibbey, K. Osathaphan, C. Saiwan, Water Res. 2006, 40, 1481.

25 F. Yu, J. Ma, S. Han, Sci. Rep. 2014, 4, 5326.

26 G. Nazari, H. Abolghasemi, M. Esmaieli, J. Taiwan Inst. Chem. E. 2016, 58, 357.

27 X. M. Sun, Y. D. Li, Chem. Eur. J. 2003, 9, 2229. 
Cite this: DOI: $10.1039 / \mathrm{c0} \times x \times 0000 x$

www.rsc.org/XXXXXX

ARTICLE TYPE

\section{The table of contents entry}

Ultralong hydrogen titanate nanobelts with hollow structure and high surface area were synthesized to serve as promising materials for effective adsorption of antibiotics from the contaminated water.

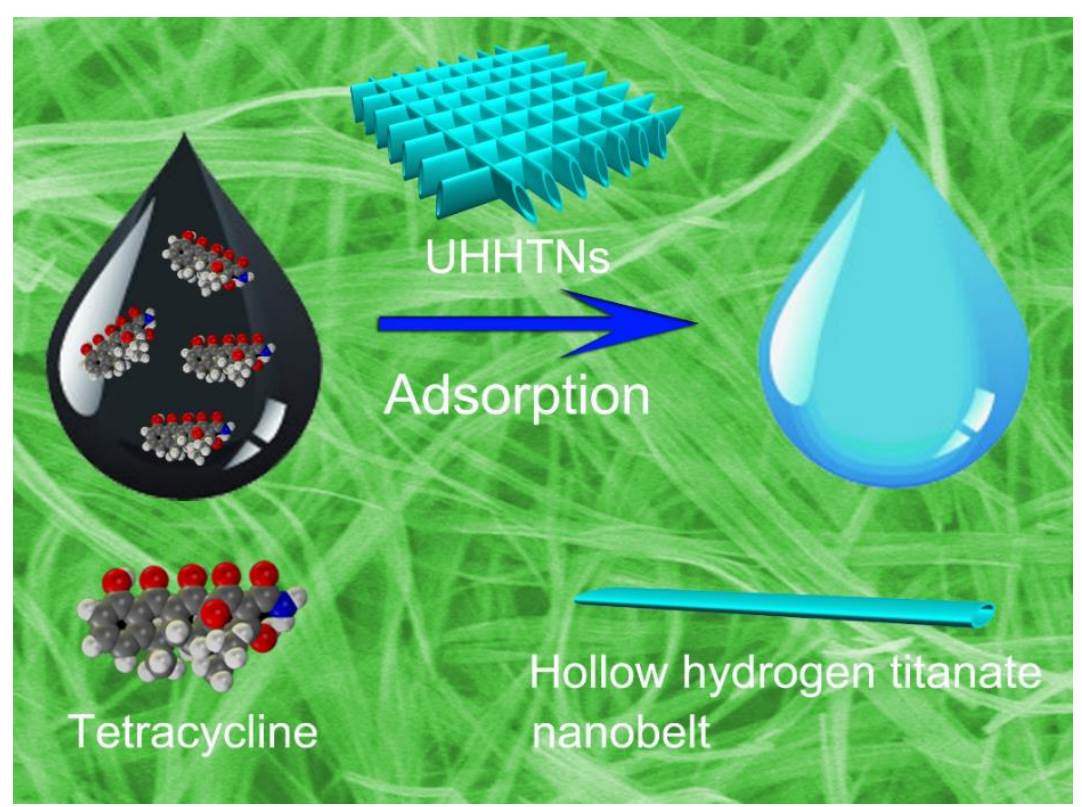

